\title{
Session 5: HIV
}

Thursday 13th November 2008. Moderator: Mirek Gorny

\section{[08.30-09.00]}

Immunoglobulin gene usage by human monoclonal antibodies that neutralize HIV-1 by targeting the envelope $V 3$ region

Miroslaw K.Gorny ${ }^{\mathrm{a}}$, Xiao-Hong Wang ${ }^{\mathrm{b}}$, Xunqing Jiang $^{\mathrm{c}}$, Constance Williams ${ }^{\mathrm{a}}$, Barbara Volsky ${ }^{\mathrm{a}}$, Kathy Revesz $^{\mathrm{b}}$, Bradley Witover ${ }^{\mathrm{a}}$, Arthur Nadas ${ }^{\mathrm{d}}$, Susan Zolla-Pazner ${ }^{\mathrm{a}, \mathrm{b}}$ and Xiang-Peng Kong ${ }^{\mathrm{c}}$

${ }^{a}$ Department of Pathology, New York University School of Medicine, New York, NY 10016, USA

${ }^{\mathrm{b}}$ Veterans Affairs New York Harbor Healthcare System, New York, NY 10010, USA

${ }^{\mathrm{c}}$ Department of Biochemistry, New York University School of Medicine, New York, NY 10016, USA

${ }^{\mathrm{d}}$ Institute of Environmental Medicine, New York University School of Medicine, New York, NY 10016, USA

Human anti-V3 monoclonal antibodies (mAbs) generated from HIV-1 infected individuals are able to cross-react with different viruses, neutralize primary isolates in in vitro experiments and prevent HIV-1 infection in animal models. Based on these data, it is suggested that V3-specific antibodies could contribute to protection against HIV-1 infection if they were induced by a vaccine in healthy individuals.

Human mAbs against the $\mathrm{V} 3$ region were produced in our laboratory from the cells of HIV-1 infected individuals using a cellular technique based on fusion of EBV-transformed lymphocytes with heteromyeloma cells. In various neutralization assays, these anti-V3 mAbs exhibit a broad range of activity which could be related to their immunogenetic background. Our studies examined the use of immunoglobulin (Ig) gene families that code for the variable region of the heavy chains of human anti-V3 mAbs. The V3 mAbs displayed a pattern of Ig gene usage different from that of mAbs to other regions of the HIV-1 envelope (anti-Env) and from $\operatorname{IgG}$ memory B cells of healthy individuals which have been reported in the published literature. There was biased usage of $\mathrm{VH}, \mathrm{D}$ and $\mathrm{JH}$ genes by
anti-V3 mAbs including preferential usage of the rarely employed VH5-51 gene segment which was detected in $35 \%$ of 51 human anti-V3 mAbs compared to only $6 \%$ in control antibodies. In contrast, human antiEnv mAbs , including those directed against the CD4binding domain, CD4-induced epitope and gp41, preferentially used the VH1-69 gene segment; none of these latter mAbs used the VH5-51 gene.Multivariate analysis also demonstrated that the usage of $\mathrm{VH}$ gene segments was significantly different between anti-V3 and anti-Env mAbs compared to Abs in normal individuals. Additional studies showed that anti-V3 mAbs preferentially used the JH3 and D2-15 genes and, to a lesser extent, the VH4-59 gene segment, while the anti-Env mAbs preferentially used D3-9 gene segments. Crystallographic analyses of two VH5-51-derived V3 Fabs complexed with $\mathrm{V} 3$ peptides revealed that the shape of the antigen binding site is determined by the $\mathrm{VH}$ gene segment. The preferential usage of several gene segments by anti-V3 mAbs may be related to conserved structural features that are unique to V3.

[09.00-09.20]

Inhibition of HIV-1 Infectivity through an innate mechanisms involving naturally occurring IgM anti-leukocyte autoantibodies

Peter I. Lobo, Kailo H. Schlegel, Wen Yuan, Gregory

C. Townsend and Jennifer A. White

Division of Nephrology and Division of Infectious Diseases and International Health, Department of Medicine, Center for Immunity Inflammation and Regenerative Medicine, and Myles $H$. Thaler Center for AIDS and Human Retrovirus Research, University of Virginia Health System, Charlottesville, VA 22908, USA

The physiological relevance of naturally occurring IgM-ALA remains to be elucidated. These autoantibodies are present from birth and increase in diverse inflammatory states that are both infectious and non- 
infectious. Clinical observations showing significantly less acute allograft rejections in recipients having high IgM-ALA levels, led us to investigate if IgM-ALA could have a functional role in attenuating $\mathrm{T}$ cell mediated inflammatory responses. In pursuit of this hypothesis, we did studies using IgM purified from the serum of normal individuals, patients with end stage renal disease, and HIV-1 infection. All preparations of IgM immunoprecipitated certain receptors e.g., CD3, CD4, CCR5, and CXCR4 from whole cell lysates but failed to immunoprecipitate IL-2R and HLA antigens. In physiological doses IgM downregulated CD4, CD2 and CD82 but not CD8 and CD28, inhibited T cell proliferation, decreased production of certain pro-inflammatory cytokines e.g. TNF- $\alpha$ IL-13 and IL-2, but not IFN- $\gamma$, IL-1B, GM-CSF, IL-6 and IL-8 and inhibited leucocyte chemotaxis. These inhibitory effects were more pronounced when using IgM from patients with high levels of IgM-ALA and these inhibitory effects were significantly reduced after using IgM pre-absorbed with leucocytes. IgM-ALA binding to leukocytes was found to be highly specific, as $<10 \%$ of IgM secreting human umbilical cord B cell clones had IgM-ALA specificity with some clones having specificity for either T cells or monocytes. Two IgM-ALA clones had specificity for CD4.

These observations prompted us to determine if IgMALA have a role in inhibiting HIV-1 infectivity principally by inhibiting viral entry into cells. We show that IgM, but not IgG, purified by size exclusion column chromatography, from individual sera of both normal and HIV-1 infected individuals is highly inhibitory $(>95 \%)$ to HIV-1 viral infectivity both in vitro using PHA+IL-2 activated PBL and in-vivo using the human PBL-SCID mouse. Inhibition was observed with physiological doses of $\operatorname{IgM}$ and even after IgM was added three days post-infection in the in-vitro assays. Absorbing IgM either with leucocytes or immobilized recombinant CD4 significantly decreased $(>80 \%)$ the inhibitory effect on HIV-1 infectivity. Inhibition was also observed with the two B cell IgM anti-CD4 clones but not with six other IgM secreting B cell clones, indicating therefore that $\operatorname{IgM}$ mediated inhibition is highly specific. IgM inhibited by $>90 \%$ syncytia formation with the X4-IIIB infected Sup T-1 cells and binding of different R5 and X4 pseudotyped viruses indicating therefore that IgM inhibits viral attachment to core-receptors. IgM from certain HIV-1 infected individuals were not inhibitory to some R5-HIV-1 viral strains indicating that certain HIV-IgM may lack antibodies reactive to strain specific co-receptor epitopes.
These data indicate that an innate immune mechanism which is present from birth i.e. IgM-ALA, has a role in inhibiting HIV-1 viral entry into cells. Developing strategies to enhance in-vivo IgM-ALA e.g. through a vaccine, could prolong the asymptomatic state in HIV1 infected individuals. The majority of this work has been published (Journal of Immunology, 2008. 180: 1769-1779).

\section{[09.20-09.50]}

Use of human monoclonal antibodies in the design of vaccines against HIV-1

Susan Zolla-Pazner

NYU School of Medicine, New York, NY 10016, USA

The past and current design of most vaccines to human pathogens is based on empiricism. One approach to the rational design of a prophylactic vaccine is: to produce human monoclonal antibodies (mAbs) that display protective activity against the pathogen in question, to identify the epitopes that they target, and, using this information, to design a vaccine that will induce a polyclonal response that targets the same epitopes. We have used this approach in the design of vaccine candidates that will induce Abs that neutralize a large proportion of the HIV-1 strains causing the current AIDS pandemic. In this effort, we have selected and characterized more than 100 human mAbs against the various epitopes of the HIV-1 envelope glycoproteins. Our initial vaccine design has focused on the Abs that target the third variable loop (V3) of the gp120 envelope glycoprotein, a neutralizing epitope of HIV-1. We will describe how we have studied 48 human anti-V3 mAbs in order to dissect the diversity of the natural human $\mathrm{Ab}$ response against V3. For this, we have derived heterohybridoma lines from the peripheral blood cells of patients infected with HIV-1 subtypes A, B or C, the three HIV-1 subtypes that cause $\sim 85 \%$ of the current pandemic. Subsequently, we have used immunogen design cycles, which consist of (a) crystallographic resolution of selected $\mathrm{V} 3 / \mathrm{mAb}$ complexes, (b) molecular modeling to extend these seed structures to apply to diverse isolates, and (c) empirical and computational methods to design immunogens that optimally focus the $\mathrm{Ab}$ response on V3. These immunogens have now moved into the testing phase to determine their ability to induce broadly neutralizing Abs in rabbits, and several sets of immunizations based on a DNA prime, protein boost protocol, demonstrate the production of polyclonal Abs that neutralize isolates from diverse HIV-1 subtypes. 
[09.50-10.20]

\section{Novel method to generate anti-HIV human monoclonal antibodies}

Mark Hicar $^{\mathrm{a}}$, Xuemin Chen ${ }^{\mathrm{a}}$, Spyros Kalams ${ }^{\mathrm{a}}$, Paul

Spearman $^{\mathrm{b}}$ and James E. Crowe, Jr. ${ }^{\mathrm{a}}$

${ }^{a}$ Departments of Pediatrics, Microbiology and

Immunology, and Medicine, Vanderbilt University,

Nashville, TN 37212, USA

${ }^{\mathrm{b}}$ Department of Pediatrics, Emory University, Atlanta, GA 30322, USA

Our laboratory has successfully developed a novel technique for isolating naturally occurring human monoclonal antibodies against protein antigens of human pathogens using single cell cloning of B cells labeled with virus-like-particles (VLPs or pseudovirions). We now have successfully adapted this technology to identify novel HIV reactive monoclonal antibodies.

Over-expression of the Gag and Env proteins inside of cells is sufficient to create pseudovirions that form native gp120 trimers on their surface and undergo budding from the cell. Vpr is a late protein of HIV that self-incorporates into developing pseudovirions. We engineered a GFP-tagged Vpr protein and showed that this chimeric protein incorporated into pseudovirions to create fluorescently labeled particles. We then mixed these fluorescent pseudovirions with selected HIV-infected subject B cells that were separated from peripheral blood mononuclear cells with magnetic bead technology. Then, using high-speed flow cytometric sorting, we selected for CD19+ (a pan B-cell marker) and GFP double-positive cells and sorted these as single cells into 96-well plates with appropriate cytokines and CD40L + feeder cells.

Immunoglobulin producing clones were identified at two weeks by ELISA and anti-HIV specificity was assessed at three weeks using recombinant gp120 and pseudovirion ELISAs. A majority of clones were reactive to trimeric gp120/gp41 rather than monomeric gp120. These clones were then harvested and RNA was extracted. RT-PCR was performed using nested primer sets that are specific for all human antibody heavy or light chain variable regions. Initial evaluation of sorted clones revealed a propensity for the use of heavy chain variable segments VH1-69 and VH1-2 by the trimer specific clones and a tremendous amount of somatic hypermutation in these clones. Clones will be expressed as Fabs and neutralization ability will be assessed and correlated with gene segment usage.

\section{[10.20-10.40]}

Structural basis of the antibody-antigen interaction in human anti-V3 HIV-1 monoclonal antibodies

Valicia Burke $^{\mathrm{a}, 1}$, Xunqiang Jiang ${ }^{\mathrm{a}}$, Constance

Williams $^{\mathrm{b}}$, Miroslaw K. Gorny ${ }^{\mathrm{b}}$, Susan

Zolla-Pazner $^{\mathrm{b}, \mathrm{c}}$ and Xiang-Peng Kong ${ }^{\mathrm{a}}$

${ }^{a}$ Department of Biochemistry, NYU School of

Medicine, New York, NY 10016, USA

${ }^{\mathrm{b}}$ Department of Pathology, NYU School of Medicine, New York, NY 10016, USA

${ }^{\mathrm{c}}$ Veterans Affairs New York Harbor Healthcare System, New York, NY 10010, USA

The third variable region (V3) of HIV-1 gp120, the extended molecular hook capable of binding the chemokine co-receptors CCR5 or CXCR4, plays a critical role in viral infectivity and tropism. V3 is also immunogenic in that immunization with gp120 often elicits neutralizing responses against V3. The availability of a large panel of human anti-V3 monoclonal antibodies (mAbs) provides a unique opportunity to systematically study the antibody-antigen interaction by structural methods. We have determined crystal structures of the antigen-binding fragment (Fab) of 7 human antiV3 mAbs, derived from patients infected with viruses from HIV-1 clades AG, B, or C, in complex with various V3 peptides. This set of atomic structures of the Fab-epitope complexes allows us to draw several general conclusions concerning these anti-V3 mAbs and the epitopes they target: i) all the epitopes are located at the crown of $\mathrm{V} 3$, a 13 amino acid motif located at the distal end of the V3 loop; ii) the epitope binding site of the $\mathrm{mAb}$ is negatively charged, reflecting the net positive charge of the V3 crown; iii) CDR H3 plays the most versatile role in epitope binding and it is primarily CDR H3 that is responsible for the induced fit; iv) the $\mathrm{mAb}$ will be promiscuous with respect to $\mathrm{V} 3$ binding if it interacts with conserved structural elements of V3. The structural information derived from these crystal structures of V3 in complex with the mAbs may help facilitate structure-based HIV vaccine designs.

${ }^{1}$ These authors contributed equally to this work. 